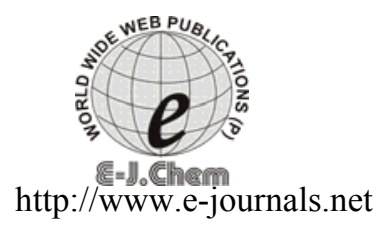

\title{
Synthesis of Some 2-Amino-5-substituted-1,3, 4-oxadiazole Derivatives in the Acetic Acid
}

\author{
SANJEEV KUMAR \\ Department of Chemistry \\ Iswar Saran Degree College (University of Allahabad) \\ Allahabad-211004, India \\ sanjivks77@gmail.com
}

Received 31 March 2011; Accepted 7 June 2011

\begin{abstract}
An environmentally benign method for the preparation of 2-amino5-substituted-1,3,4-oxadiazoles (1a-n) at platinum electrode through the electrooxidation of semicarbazone at room temperature is reported. The electrolysis were carried out at controlled potential in acetic acid solvent using lithium perchlorate as supporting electrolyte in an undivided cell assembly. Square shaped plates of platinum were used for working as well as counter electrode and a third electrode saturated calomel electrode was used as a reference electrode for the measurement of potential difference between two electrodes during the electrolysis.
\end{abstract}

Keywords: Semicarbazone, 2-Amino-1,3,4-oxadiazoles, Electrooxidation, Controlled potential, Electrolysis

\section{Introduction}

2-Amino-5-substituted-1,3,4-oxadiazoles are of considerable pharmaceutical and material interest, which is documented by steadily increasing numbers of publications and patents. 1,3,4-Oxadiazoles have been found to exhibit diverse biological activities such as antibacterial ${ }^{1-3}$, anti-HIV $^{1-3}$, anti-microbial ${ }^{1-3}$, anti-fungal ${ }^{4,5}$, anti-inflammatory ${ }^{6}$, hypotensive ${ }^{7}$, muscle relaxant ${ }^{8}$ and antimitotic activity'.

Methods for the synthesis of oxadiazoles ${ }^{10-14}$ (1) include bromine oxidation of semicarbazide derivative and the cyclodesulfurisation of acylthiosemicarbazide derivative in solution using $\mathrm{I}_{2} / \mathrm{NaOH}$ or 1,3 -dicyclohexylcarbodimide (DCC) ${ }^{15-19}$ as well as mercury (II) acetate $\left[\mathrm{Hg}(\mathrm{OAc})_{2}\right]$ or yellow mercury (II) oxide $\mathrm{HgO}^{20-22}$. All these methods are usually carried out in various different synthetic steps and require heating. The handling of these reagents is not only difficult but also hazardous to the environment. Each stage of the reaction including extraction and purification of the products from the mixture requires great precautions. Evans ${ }^{23}$ has synthesized a similar cyclized product by rapid parallel synthesis in efficient one-pot preparation using resin-bound reagents. 


\section{Experimental}

The purity of the synthesized compounds were ascertained by thin layer chromatography (TLC) on silica gel G $60 \mathrm{~F}_{254}$ in various solvent systems using iodine vapor to stain products as detecting agent. All melting points were determined in open capillaries and were uncorrected. Infra red spectra were recorded on a Shimadzu 8201 PC IR spectrophotometer in $\mathrm{KBr}$ pellets and reported in $\mathrm{cm}^{-1}$. ${ }^{1} \mathrm{H}$ NMR and ${ }^{13} \mathrm{C}$ NMR spectra were measured at room temperature on Bruker DRX $300 \mathrm{MHz}$ FT spectrometers, with tetramethylsilane (TMS) and $\mathrm{CDCl}_{3}$ or $\mathrm{C}_{6} \mathrm{D}_{6}$ as internal standards. Carbon multiplities were assigned by DEPT techniques. The signals are described as singlet $(s)$, doublet $(d)$, triplet $(t)$, quartet $(q)$ and multiplet $(m)$. Microanalyses were carried out in the Elementar Vario EL III.

\section{General procedure for the preparation of 2-amino-5-substituted-1,3,4-oxadiazoles (1a-n)}

Semicarbazide hydrochloride (3) (2.0 g, $17.92 \mathrm{mmol})$ and sodium acetate (1.0 g, $12.19 \mathrm{mmol})$ was dissolved in $20 \mathrm{~mL}$ water. Then aldehyde (2) $(1.0 \mathrm{~g}, 6.08 \mathrm{mmol})$ was added with continuous stirring. The mixture was left overnight, which gave semicarbazone (4) as a solid for the electrolysis. Semicarbazone (4) $(2.0 \mathrm{~g}, 10.12 \mathrm{mmol})$ was dissolved in $200 \mathrm{~mL}$ acetic acid and lithium perchlorate $(0.106 \mathrm{~g}, 0.76 \mathrm{mmol})$ was dissolved in the above solution to maintain the strength of supporting electrolyte $0.001 \mathrm{M}$.

\section{Electrolysis}

Preparative scale controlled potential electrolysis ${ }^{24-28}$ was performed at room temperature in $250 \mathrm{~mL}$ three-electrode cell assembly with platinum plate as working as well as counter electrode and saturated calomel electrode (SCE) as reference electrode. Magnetic stirrer was used for efficient mixing of reaction mixture. Solid products formed on electrode do not adhere to the electrode surface due to continuous stirring. All the electrolysis experiments were carried out at their corresponding oxidation potentials and were completed in 3 to $5 \mathrm{~h}$. After which no oxidation product was seen to diffuse in the bulk. All the products were solid and coloured and entirely different from the starting compound. The current potential data was recorded with the help of a potentiostat at intervals of $15 \mathrm{~min}$ (Table 1). Approximately $4.3 \times 10^{5}-5.8 \times 10^{5} \mathrm{C} \mathrm{mol}^{-1}$ of electricity was passed for the electrolysis.

Table 1. Current, potential and yield data for synthesis of 1,3,4-oxadiazoles (1a-n)

\begin{tabular}{ccccccc}
\hline \multirow{2}{*}{ Entry } & \multirow{2}{*}{$\mathrm{R}$} & Compd. & $\begin{array}{c}\text { Time, } \\
\mathrm{h}\end{array}$ & $\begin{array}{c}\text { Potential, } \\
\mathrm{mV}\end{array}$ & $\begin{array}{c}\text { Current, } \\
\mathrm{mA}\end{array}$ & $\begin{array}{c}\text { Yield, } \\
\%\end{array}$ \\
\hline 1 & $4-\mathrm{ClC}_{6} \mathrm{H}_{4}$ & $\mathbf{1 a}$ & 3 & 2030 & 110 & 82 \\
2 & $4-\mathrm{FC}_{6} \mathrm{H}_{4}$ & $\mathbf{1 b}$ & 5 & 2320 & 150 & 86 \\
3 & $4-\left(\mathrm{NO}_{2}\right) \mathrm{C}_{6} \mathrm{H}_{4}$ & $\mathbf{1 c}$ & 4 & 2500 & 170 & 85 \\
4 & $4-\left(\mathrm{CH}_{3} \mathrm{CONH}_{4}\right) \mathrm{C}_{6} \mathrm{H}_{4}$ & $\mathbf{1 d}$ & 3 & 1350 & 140 & 90 \\
5 & $\mathrm{C}_{7} \mathrm{H}_{15}$ & $\mathbf{1 e}$ & 4 & 2100 & 125 & 87 \\
6 & $4-\mathrm{Me}_{2} \mathrm{NC}_{6} \mathrm{H}_{4} \mathrm{CHCH}$ & $\mathbf{1 f}$ & 4 & 1900 & 150 & 88 \\
7 & $\left(\mathrm{C}_{6} \mathrm{H}_{5} \mathrm{CO}\right) \mathrm{C}_{6} \mathrm{H}_{4}$ & $\mathbf{1 g}$ & 5 & 2000 & 170 & 80 \\
8 & $3-\mathrm{C}_{6} \mathrm{H}_{5} \mathrm{CO}, 2-(\mathrm{OMe}) \mathrm{C}_{6} \mathrm{H}_{3}$ & $\mathbf{1 h}$ & 5 & 1750 & 160 & 81 \\
9 & $4-\left(\mathrm{C}_{4} \mathrm{H}_{9} \mathrm{O}\right) \mathrm{C}_{6} \mathrm{H}_{5}$ & $\mathbf{1 i}$ & 3 & 1800 & 190 & 90 \\
10 & $3,4-(\mathrm{OMe})_{2} \mathrm{C}_{6} \mathrm{H}_{3}$ & $\mathbf{1 j}$ & 4 & 1660 & 110 & 87 \\
11 & $4-(\mathrm{AcO}) \mathrm{C}_{6} \mathrm{H}_{4}$ & $\mathbf{1 k}$ & 3 & 1540 & 130 & 95 \\
12 & $2,4-\mathrm{F}_{2} \mathrm{C}_{6} \mathrm{H}_{3}$ & $\mathbf{1 l}$ & 5 & 2220 & 160 & 77 \\
13 & $5-\mathrm{Br}, 2-(\mathrm{OMe}) \mathrm{C}_{6} \mathrm{H}_{3}$ & $\mathbf{1 m}$ & 5 & 2100 & 130 & 79 \\
14 & $2-\mathrm{Cl}, 6-\left(\mathrm{NO}_{2}\right) \mathrm{C}_{6} \mathrm{H}_{3}$ & $\mathbf{1 n}$ & 5 & 2250 & 170 & 81 \\
\hline
\end{tabular}


The products were extracted from the acetic acid solution to chloroform by the simple solvent extraction technique. The two-immiscible layers of acetic acid and chloroform were shaken in a separatory funnel and allowed to settle down. After some time the chloroform layer containing the product oxadiazole, was removed. The extracted chloroform layer was separate out with the help of rotatory evaporator and oxadiazole (1a-n) were obtained in the excellent yield and purity was checked by the TLC. The product oxadiazole can also be separate out from the reaction solution with the help of column chromatography without using chloroform. This is a more time taking process in comparison to extraction process.

\section{The physical and spectral data of the compounds (1a-n)}

\section{2-Amino-5-(4-chlorophenyl)-1,3,4-oxadiazole (1a)}

Brownish crystals, m.p. 81-83 ${ }^{\circ} \mathrm{C}$, IR (KBr, cm $\left.{ }^{-1}\right) 3360(\mathrm{NH}), 3045(\mathrm{ArC}-\mathrm{H}), 1610(\mathrm{C}=\mathrm{N}), 1265$, 1070 (C-O-C), 600-800 (Ar-Cl), 860, 750 (substituted benzene), ${ }^{1} \mathrm{H}$ NMR (DMSO, $\mathrm{d}_{6}, \delta / \mathrm{ppm}$ ): $7.75\left(\mathrm{~s}, 2 \mathrm{H}, \mathrm{NH}_{2}\right), 7.71\left(\mathrm{~d}, 2 \mathrm{H}, \mathrm{Ar}-\mathrm{H}_{2 ; 6}, J=8.6 \mathrm{~Hz}\right), 7.61\left(\mathrm{~d}, 2 \mathrm{H}, \mathrm{Ar}-\mathrm{H}_{3 ; 5}, J=8.6 \mathrm{~Hz}\right),{ }^{13} \mathrm{C} \mathrm{NMR}$ (DMSO, $\left.\mathrm{d}_{6}, \delta / \mathrm{ppm}\right): 170.2,148.5,141.8,140.6,139.5,129.5,128.7,126.5$. Anal. calcd. for $\mathrm{C}_{8} \mathrm{H}_{6} \mathrm{~N}_{3} \mathrm{OCl}: \mathrm{C}, 49.10 ; \mathrm{H}, 3.06 ; \mathrm{N}, 21.48 ; \mathrm{Cl}, 18.15$. Found: C, 48.95; H, 3.00; N, 21.20; Cl, $17.98 \%$.

\section{2-Amino-5-(4-fluorophenyl)-1,3,4-oxadiazole (1b)}

Brown needles, m.p. 76-78 ${ }^{0} \mathrm{C}$, IR (KBr, cm $\left.{ }^{-1}\right) 3360(\mathrm{NH}), 3055$ (ArC-H), $1604(\mathrm{C}=\mathrm{N}), 1255$, 1080 (C-O-C), 1060 (Ar-F), 950, 860, 740 (substituted benzene), ${ }^{1} \mathrm{H}$ NMR (DMSO, $\mathrm{d}_{6}, \delta / \mathrm{ppm}$ ): 7.75 (s, 2H, NH $\left.\mathrm{N}_{2}\right), 7.21$ (m, 2H, Ar- $\mathrm{H}_{2 ; 6}$ ), 7.38 (m, 2H, Ar- $\left.\mathrm{H}_{3 ; 5}\right),{ }^{13} \mathrm{C}$ NMR (DMSO, $\mathrm{d}_{6}, \delta / \mathrm{ppm}$ ): $173.1,163.7\left(\mathrm{~d}, J_{\mathrm{C}-\mathrm{F}}=250 \mathrm{~Hz}, \mathrm{ArC}_{4}\right), 147.7,147.2,137.5,132.3\left(\mathrm{~d}, J_{\mathrm{C}-\mathrm{F}}=8.6 \mathrm{~Hz}, \mathrm{ArC}_{2 ; 6}\right), 122.4$ $\left(\mathrm{d}, J_{\mathrm{C}-\mathrm{F}}=3.6 \mathrm{~Hz}, \mathrm{ArC}_{1}\right), 116.3\left(\mathrm{~d}, J_{\mathrm{C}-\mathrm{F}}=22 \mathrm{~Hz}, \mathrm{ArC}_{3 ; 5}\right)$. Anal. calcd. for $\mathrm{C}_{8} \mathrm{H}_{6} \mathrm{~N}_{3} \mathrm{OF}: \mathrm{C}, 53.63$; H, 3.35; N, 23.46; F, 10.05. Found: C, 53.25; H, 3.25; N, 23.20; F, 9.95\%.

\section{2-Amino-5-(4-nitrophenyl)-1,3,4-oxadiazole (1c)}

Dark yellow needles, m.p. 81-83 ${ }^{0} \mathrm{C}$, IR $\left(\mathrm{KBr}, \mathrm{cm}^{-1}\right) 3341(\mathrm{NH}), 3035(\mathrm{ArC}-\mathrm{H}), 1609(\mathrm{C}=\mathrm{N})$, $1580\left(\mathrm{Ar}^{-\mathrm{NO}_{2}}\right), 1272,1065$ (C-O-C), 890, 750, 690 (substituted benzene), ${ }^{1} \mathrm{H}$ NMR (DMSO, $\mathrm{d}_{6}$, $\delta / \mathrm{ppm}): 7.75\left(\mathrm{~s}, 2 \mathrm{H}, \mathrm{NH}_{2}\right), 7.71\left(\mathrm{~d}, 2 \mathrm{H}, \mathrm{Ar}-\mathrm{H}_{2 ; 6}, J=8.6 \mathrm{~Hz}\right), 7.62\left(\mathrm{~d}, 2 \mathrm{H}, \mathrm{Ar}-\mathrm{H}_{3 ; 5}, J=8.6 \mathrm{~Hz}\right)$, ${ }^{13} \mathrm{C}$ NMR (DMSO, $\mathrm{d}_{6}, \delta / \mathrm{ppm}$ ): 171.5, 148.1, 147.2, 141.2, 136.4, 134.2, 129.7, 123.7. Anal. calcd. for $\mathrm{C}_{8} \mathrm{H}_{6} \mathrm{~N}_{4} \mathrm{O}_{3}$ : C, 46.60; H, 2.91; N, 27.18. Found: C, 46.10; H, 2.85; N, 26.80\%.

\section{2-Amino-5-(4-acetamidophenyl)-1,3,4-oxadiazole (1d)}

Dark brownish needles, m.p. $78{ }^{0} \mathrm{C}$, IR $\left(\mathrm{KBr}, \mathrm{cm}^{-1}\right)$ 3460-3500 $\left(\mathrm{CO}-\mathrm{NH}_{2}\right), 3444(\mathrm{NH}), 2927$ (Ar C-H), 2856 (aliphatic C-H), $1611(\mathrm{C}=\mathrm{N}), 1270(\mathrm{C}=\mathrm{O})$, 1250, 1062 (C-O-C), 915, 870, 675 (substituted benzene), ${ }^{1} \mathrm{H}$ NMR (DMSO, $\left.\mathrm{d}_{6}, \delta / \mathrm{ppm}\right): 8.13(\mathrm{~s}, 1 \mathrm{H}, \mathrm{CONH}), 7.75\left(\mathrm{~s}, 1 \mathrm{H}, \mathrm{NH}_{2}\right.$ ), $7.70\left(\mathrm{~d}, 2 \mathrm{H}, \mathrm{Ar}-\mathrm{H}_{2 ; 6}, J=8.6 \mathrm{~Hz}\right), 7.61\left(\mathrm{~d}, 2 \mathrm{H}, \mathrm{Ar}-\mathrm{H}_{3 ; 5}, J=8.6 \mathrm{~Hz}\right), 1.92\left(\mathrm{~s}, 3 \mathrm{H}, \mathrm{CH}_{3}\right),{ }^{13} \mathrm{C} \mathrm{NMR}$ (DMSO, $\mathrm{d}_{6}, \delta / \mathrm{ppm}$ ): 172.5, 169.5, 147.5, 140.2, 138.9, 128.4, 122.8, 118.6, 118.5, 20.6. Anal. calcd. for $\mathrm{C}_{10} \mathrm{H}_{10} \mathrm{~N}_{4} \mathrm{O}_{2}$ : C, 55.06; H, 4.58; N, 25.68. Found: C, 54.80; H, 4.43; N, 25.52\%.

\section{2-Amino-5-heptyl-1,3,4-oxadiazole (1e)}

Light yellow crystals, m.p. $83{ }^{\circ} \mathrm{C}$, IR $\left(\mathrm{KBr}, \mathrm{cm}^{-1}\right) 3360(\mathrm{NH}), 3060(\mathrm{C}-\mathrm{H}), 1608(\mathrm{C}=\mathrm{N}), 1280$, 1066 (C-O-C), ${ }^{1} \mathrm{H}$ NMR (DMSO, d 6 , $\left.\delta / \mathrm{ppm}\right): 7.75$ (s, 2H, NH $\mathrm{NH}_{2}, 3.5$ (t, $2 \mathrm{H}, \mathrm{CH}_{2}\left(\mathrm{CH}_{2}\right)_{5} \mathrm{CH}_{3}$, $J=7.3 \mathrm{~Hz}$ ), 1.5 (sextet, $2 \mathrm{H}, \mathrm{CH}_{2}\left(\mathrm{CH}_{2}\right)_{4} \mathrm{CH}_{2} \mathrm{CH}_{3}, J=7.3 \mathrm{~Hz}$ ), 1.2-1.8 (quintet, $8 \mathrm{H}, \mathrm{CH}_{2}$ $\left.\left(\mathrm{CH}_{2}\right)_{4} \mathrm{CH}_{2} \mathrm{CH}_{3}, J=7.3 \mathrm{~Hz}\right), 0.9\left(\mathrm{t}, 3 \mathrm{H}, \mathrm{CH}_{2}\left(\mathrm{CH}_{2}\right)_{5} \mathrm{CH}_{3}, J=7.3 \mathrm{~Hz}\right),{ }^{13} \mathrm{C}$ NMR (DMSO, $\mathrm{d}_{6}$, $\delta / \mathrm{ppm}): 170.8,148.6,147.6,32.2,32.1,23.5,23.3,23.1,14.1$. Anal. calcd. for $\mathrm{C}_{9} \mathrm{H}_{17} \mathrm{~N}_{3} \mathrm{O}: \mathrm{C}$, 59.01; H, 9.28; N, 22.95. Found: C, 58.76; H, 9.15; N, 22.60\%. 


\section{2-Amino-5-(4-N,N-dimethylaminocinnamyl)-1,3,4-oxadiazole (1f)}

Dark blackish crystals, m.p. $71-73{ }^{0} \mathrm{C}$, IR $\left(\mathrm{KBr}, \mathrm{cm}^{-1}\right) 3336(\mathrm{NH}), 3033(=\mathrm{C}-\mathrm{H}), 3030(\mathrm{ArC}-\mathrm{H})$, 2855 (aliphatic $\mathrm{C}-\mathrm{H}), 1670(\mathrm{C}=\mathrm{C}), 1606(\mathrm{C}=\mathrm{N}), 1262,1067(\mathrm{C}-\mathrm{O}-\mathrm{C}), 920,860,790$ (substituted benzene), ${ }^{1} \mathrm{H}$ NMR (DMSO, $\left.\mathrm{d}_{6}, \delta / \mathrm{ppm}\right): 7.75$ (s, $\left.2 \mathrm{H}, \mathrm{NH}_{2}\right), 7.71\left(\mathrm{~d}, 2 \mathrm{H}, \mathrm{Ar}-\mathrm{H}_{2 ; 6}, J=8.6 \mathrm{~Hz}\right)$, $7.61\left(\mathrm{~d}, 2 \mathrm{H}, \mathrm{Ar}-\mathrm{H}_{3 ; 5}, J=8.6 \mathrm{~Hz}\right), 6.52(\mathrm{~d}, 1 \mathrm{H}, \mathrm{PhCH}=\mathrm{CH}, J=16.8 \mathrm{~Hz}), 6.39(\mathrm{~d}, 1 \mathrm{H}, \mathrm{PhCH}=\mathrm{CH}$, $J=16.8 \mathrm{~Hz}), 2.35\left(\mathrm{~s}, 6 \mathrm{H}, \mathrm{CH}_{3}\right),{ }^{13} \mathrm{C}$ NMR (DMSO, $\left.\mathrm{d}_{6}, \delta / \mathrm{ppm}\right): 170.6,147.3,146.5,138.1$, 137.6, 129.2, 129.1, 126.9, 126.5, 14.2, 13.7. Anal. calcd. for $\mathrm{C}_{12} \mathrm{H}_{14} \mathrm{~N}_{4} \mathrm{O}: \mathrm{C}, 62.60 ; \mathrm{H}, 6.08 ; \mathrm{N}$, 24.34. Found: C, 62.50; H, 5.90; N, 24.25\%.

\section{2-Amino-5-(2-benzoylphenyl)-1,3,4-oxadiazole (1g)}

Brownish needles, m.p. $70-72{ }^{\circ} \mathrm{C}$, IR $\left(\mathrm{KBr}, \mathrm{cm}^{-1}\right) 3350(\mathrm{NH}), 3045(\mathrm{ArC}-\mathrm{H}), 1755(\mathrm{C}=\mathrm{O}), 1620$ $(\mathrm{C}=\mathrm{N}), 1273,1059$ (C-O-C), 880, 820, 785 (substituted benzene), ${ }^{1} \mathrm{H}$ NMR (DMSO, $\mathrm{d}_{6}, \delta / \mathrm{ppm}$ ): $7.98(\mathrm{~d}, 2 \mathrm{H}, \mathrm{Ar}-\mathrm{H}, J=8.6 \mathrm{~Hz}), 6.93-7.03(\mathrm{~m}, 2 \mathrm{H}, \mathrm{Ar}-\mathrm{H}), 7.75\left(\mathrm{~s}, 2 \mathrm{H}, \mathrm{NH}_{2}\right), 7.45-7.56(\mathrm{~m}, 3 \mathrm{H}$, Ar-H), 7.38-7.42 (m, 2H, Ar-H), ${ }^{13} \mathrm{C}$ NMR (DMSO, $\left.\mathrm{d}_{6}, \delta / \mathrm{ppm}\right): 171.2,168.6,149.5,137.1$, $136.3,132.9,131.3,130.2,128.7,128.5,128.1,128.0,127.8$. Anal. calcd. for $\mathrm{C}_{15} \mathrm{H}_{11} \mathrm{~N}_{3} \mathrm{O}_{2}: \mathrm{C}$, 67.92; H, 4.15; N, 15.84. Found: C, 67.60; H, 4.10; N, 15.65\%.

\section{2-Amino-5-(3-benzoyl-2-methoxyphenyl)-1,3,4-oxadiazole (1 h)}

Brown crystals, m.p. $75-76{ }^{0} \mathrm{C}$, IR $\left(\mathrm{KBr}, \mathrm{cm}^{-1}\right) 3261(\mathrm{NH}), 3045$ (ArC-H), $2815\left(\mathrm{OCH}_{3}\right), 1760$ $(\mathrm{C}=\mathrm{O}), 1609(\mathrm{C}=\mathrm{N}), 1270,1069(\mathrm{C}-\mathrm{O}-\mathrm{C}), 915,870,790$ (substituted benzene), ${ }^{1} \mathrm{H}$ NMR (DMSO, $\left.\mathrm{d}_{6}, \delta / \mathrm{ppm}\right): 8.75(\mathrm{~s}, 1 \mathrm{H}, \mathrm{Ar}-\mathrm{H}), 7.98(\mathrm{~d}, 2 \mathrm{H}, \mathrm{Ar}-\mathrm{H}, \mathrm{J}=8.6 \mathrm{~Hz}), 7.75\left(\mathrm{~s}, 2 \mathrm{H}, \mathrm{NH}_{2}\right), 7.45-$ $7.56(\mathrm{~m}, 3 \mathrm{H}, \mathrm{Ar}-\mathrm{H}), 6.95-7.41(\mathrm{~m}, 3 \mathrm{H}, \mathrm{Ar}-\mathrm{H}), 6.93-7.03(\mathrm{~m}, 3 \mathrm{H}, \mathrm{Ar}-\mathrm{H}), 3.11\left(\mathrm{~s}, 3 \mathrm{H}, \mathrm{OCH}_{3}\right),{ }^{13} \mathrm{C}$ NMR (DMSO, $\left.\mathrm{d}_{6}, \delta / \mathrm{ppm}\right): 172.6,168.7,147.5,137.1,136.3,133.1,132.9,131.3,129.5,128.5$, 128.2, 128.0, 127.8, 114.6, 52.3. Anal. calcd. for $\mathrm{C}_{16} \mathrm{H}_{13} \mathrm{~N}_{3} \mathrm{O}_{3}$ : C, 65.08; H, 4.90; N, 14.23. Found: C, 64.88; H, 4.57; N, 14.15\%.

\section{2-Amino-5-(4-butoxyphenyl)-1,3,4-oxadiazole (1i)}

Yellow crystals, m.p. $69-71{ }^{0} \mathrm{C}$, IR $\left(\mathrm{KBr}, \mathrm{cm}^{-1}\right) 3261(\mathrm{NH}), 3060(\mathrm{C}-\mathrm{H}), 2820\left(\mathrm{OC}_{4} \mathrm{H}_{9}\right), 1608$ $(\mathrm{C}=\mathrm{N}), 1269,1067$ (C-O-C), 950, 890, 770 (substituted benzene), ${ }^{1} \mathrm{H}$ NMR (DMSO, $\mathrm{d}_{6}, \delta / \mathrm{ppm}$ ): $7.75\left(\mathrm{~s}, 2 \mathrm{H}, \mathrm{NH}_{2}\right), 7.71\left(\mathrm{~d}, 2 \mathrm{H}, \mathrm{Ar}-\mathrm{H}_{2 ; 6}, J=8.6 \mathrm{~Hz}\right), 7.63\left(\mathrm{~d}, 2 \mathrm{H}, \mathrm{Ar}-\mathrm{H}_{3 ; 5}, J=8.6 \mathrm{~Hz}\right), 3.8(\mathrm{t}, 2 \mathrm{H}$, $\mathrm{CH}_{2}\left(\mathrm{CH}_{2}\right)_{2} \mathrm{CH}_{3} J=7.3 \mathrm{~Hz}$ ), 2.3 (Quintet, $2 \mathrm{H}, \mathrm{CH}_{2} \mathrm{CH}_{2} \mathrm{CH}_{2} \mathrm{CH}_{3}, J=7.3 \mathrm{~Hz}$ ), 1.6 (sextet, $2 \mathrm{H}$, $\mathrm{CH}_{2} \mathrm{CH}_{2} \mathrm{CH}_{2} \mathrm{CH}_{3}, J=7.3 \mathrm{~Hz}$ ), 0.9 (t, $3 \mathrm{H}, \mathrm{CH}_{2} \mathrm{CH}_{2} \mathrm{CH}_{2} \mathrm{CH}_{3}, J=7.3 \mathrm{~Hz}$ ), ${ }^{13} \mathrm{C}$ NMR (DMSO, $\mathrm{d}_{6}$, $\delta / \mathrm{ppm})$ : 172.3, 149.9, 142.8, 129.5, 128.6, 127.6, 114.2, 114.1, 65.6, 54.5, 23.8, 10.0. Anal. calcd. for $\mathrm{C}_{12} \mathrm{H}_{15} \mathrm{~N}_{3} \mathrm{O}_{2}$ : C, 61.80; H, 6.43; N, 18.02. Found: C, 61.45; H, 6.23; N, $17.95 \%$.

\section{2-amino-5-(3,4-dimethoxyphenyl)-1,3,4-oxadiazole (1j)}

Dark brownish needles, m.p. 77-79 ${ }^{0} \mathrm{C}$, IR $\left(\mathrm{KBr}, \mathrm{cm}^{-1}\right) 3444$ (NH), 2927 (Ar C-H), 2856 (aliphatic C-H), $2865\left(\mathrm{O}-\mathrm{CH}_{3}\right), 1605(\mathrm{C}=\mathrm{N}), 1268,1074(\mathrm{C}-\mathrm{O}-\mathrm{C}), 895,860,790$ (substituted benzene), ${ }^{1} \mathrm{H}$ NMR (DMSO, $\left.\mathrm{d}_{6}, \delta / \mathrm{ppm}\right): 8.76(\mathrm{~s}, 1 \mathrm{H}, \mathrm{Ar}-\mathrm{H}), 7.91(\mathrm{~d}, 1 \mathrm{H}, \mathrm{Ar}-\mathrm{H}, J=8.2 \mathrm{~Hz}), 7.75$ $\left(\mathrm{s}, 2 \mathrm{H}, \mathrm{NH}_{2}\right), 7.30(\mathrm{~d}, 1 \mathrm{H}, \mathrm{Ar}-\mathrm{H}, J=8.2 \mathrm{~Hz}), 3.10-3.73\left(\mathrm{~s}, 6 \mathrm{H}, \mathrm{OCH}_{3}\right),{ }^{13} \mathrm{C}$ NMR $\left(\mathrm{DMSO}, \mathrm{d}_{6}\right.$, $\delta / \mathrm{ppm})$ : 171.7, 148.8, 143.9, 143.4, 136.6, 133.7, 121.8, 116.5, 54.1, 46.7. Anal. calcd. for $\mathrm{C}_{10} \mathrm{H}_{11} \mathrm{~N}_{3} \mathrm{O}_{3}$ : C, 54.29; H, 4.97; N, 19.00 . Found: C, 54.00; H, 4.73; N, 18.75\%.

\section{2-amino-5-(4-acetoxyphenyl)-1,3,4-oxadiazole (1k)}

Light brown crystals, m.p. 78-79 ${ }^{0} \mathrm{C}$, IR $\left(\mathrm{KBr}, \mathrm{cm}^{-1}\right) 3330(\mathrm{NH}), 3045$ (ArC-H), 2855 (aliphatic $\mathrm{C}-\mathrm{H}), 1720(\mathrm{C}=\mathrm{O}), 1607(\mathrm{C}=\mathrm{N}), 1285(\mathrm{C}-\mathrm{O}), 1265,1069(\mathrm{C}-\mathrm{O}-\mathrm{C}), 910,860,735$ (substituted benzene), ${ }^{1} \mathrm{H}$ NMR (DMSO, $\left.\mathrm{d}_{6}, \delta / \mathrm{ppm}\right): 7.75\left(\mathrm{~s}, 2 \mathrm{H}, \mathrm{NH}_{2}\right.$ ), $7.72\left(\mathrm{~d}, 2 \mathrm{H}, \mathrm{Ar}-\mathrm{H}_{2 ; 6}, J=8.6 \mathrm{~Hz}\right.$ ), 
$7.63\left(\mathrm{~d}, 2 \mathrm{H}, \mathrm{Ar}-\mathrm{H}_{3 ; 5}, J=8.6 \mathrm{~Hz}\right), 1.48\left(\mathrm{~s}, 3 \mathrm{H}, \mathrm{CH}_{3}\right),{ }^{13} \mathrm{C} \mathrm{NMR}\left(\mathrm{DMSO}, \mathrm{d}_{6}, \delta / \mathrm{ppm}\right): 172.2,165.3$, $146.5,137.8,136.5,128.8,127.8,126.5,54.5,20.6$. Anal. calcd. for $\mathrm{C}_{10} \mathrm{H}_{9} \mathrm{~N}_{3} \mathrm{O}_{3}: \mathrm{C}, 54.79 ; \mathrm{H}$, 4.10; N, 19.17. Found: C, 53.94; H, 4.05; N, 19.10\%.

\section{2-Amino-5-(2,4-difluorophenyl)-1,3,4-oxadiazole (11)}

Dark brownish crystals, m.p. 80-82 ${ }^{\circ} \mathrm{C}$, IR $\left(\mathrm{KBr}, \mathrm{cm}^{-1}\right) 3360(\mathrm{NH}), 3050(\mathrm{ArC}-\mathrm{H}), 1615(\mathrm{C}=\mathrm{N})$, 1259, 1068 (C-O-C), 1100-1400 (Ar-F), 940, 870, 770 (substituted benzene), ${ }^{1} \mathrm{H}$ NMR (DMSO, $\left.\mathrm{d}_{6}, \delta / \mathrm{ppm}\right): 8.77$ (s, $\left.1 \mathrm{H}, \mathrm{Ar}-\mathrm{H}\right), 7.75\left(\mathrm{~s}, 2 \mathrm{H}, \mathrm{NH}_{2}\right), 7.15-7.47$ (m, 2H, Ar-H) ${ }^{13} \mathrm{C}$ NMR (DMSO, $\mathrm{d}_{6}$, $\delta / \mathrm{ppm}): 171.8,164.9,149.5,142.5,139.4,125.6,122.2,116.5,100.1$. Anal. calcd. for $\mathrm{C}_{8} \mathrm{H}_{5} \mathrm{~N}_{3} \mathrm{OF}_{2}: \mathrm{C}$, 48.73; H, 2.53; N, 21.31; F, 19.28. Found: C, 48.67; H, 2.40; N, 21.10; F, 19.05\%.

\section{2-amino-5-(5-bromo-2-methoxyphenyl)-1,3,4-oxadiazole (1 m)}

Brownish crystals, m.p. $75-77{ }^{0} \mathrm{C}$, IR $\left(\mathrm{KBr}, \mathrm{cm}^{-1}\right) 3360(\mathrm{NH}), 3045$ (ArC-H), 2860 (aliphatic $\mathrm{C}-\mathrm{H}), 2820\left(\mathrm{O}-\mathrm{CH}_{3}\right), 1613(\mathrm{C}=\mathrm{N}), 1265,1072(\mathrm{C}-\mathrm{O}-\mathrm{C}), 980,890,750,595$ (substituted benzene), ${ }^{1} \mathrm{H}$ NMR (DMSO, $\left.\mathrm{d}_{6}, \delta / \mathrm{ppm}\right): 8.78$ (s, 1H, Ar-H), 7.89 (d, 1H, Ar-H, J=8.2 Hz), 7.75 (s, $\left.2 \mathrm{H}, \mathrm{NH}_{2}\right), 7.28$ (d, $\left.1 \mathrm{H}, \mathrm{Ar}-\mathrm{H}, J=8.2 \mathrm{~Hz}\right), 3.11\left(\mathrm{~s}, 3 \mathrm{H}, \mathrm{OCH}_{3}\right),{ }^{13} \mathrm{C}$ NMR $\left(\mathrm{DMSO}, \mathrm{d}_{6}, \delta / \mathrm{ppm}\right)$ : $172.2,158.9,147.7,140.6,132.9,125.1,115.4,106.4,53.8$. Anal. calcd. for $\mathrm{C}_{9} \mathrm{H}_{8} \mathrm{~N}_{3} \mathrm{O}_{2} \mathrm{Br}: \mathrm{C}$, 40.00; H, 2.96; N, 15.55; Br, 29.62. Found: C, 39.52; H, 2.46; N, 15.30; Br, 29.56\%.

\section{2-Amino-5-(2-chloro-6-nitrophenyl)-1,3,4-oxadiazole (1n)}

Dark yellowish needles, m.p. $71-73{ }^{0} \mathrm{C}$, IR $\left(\mathrm{KBr}, \mathrm{cm}^{-1}\right) 3341(\mathrm{NH}), 3035(\mathrm{ArC}-\mathrm{H}), 1607(\mathrm{C}=\mathrm{N})$, $1580\left(\mathrm{Ar}-\mathrm{NO}_{2}\right), 1275,1070$ (C-O-C), 985, 865, 810, 730 (substituted benzene), ${ }^{1} \mathrm{H}$ NMR (DMSO, $\left.\mathrm{d}_{6}, \delta / \mathrm{ppm}\right): 7.75$ (s, 2H, NH $\mathrm{N}_{2}$ ) 7.05-7.19 (m, 3H, Ar-H), ${ }^{13} \mathrm{C}$ NMR (DMSO, $\mathrm{d}_{6}, \delta / \mathrm{ppm}$ ): 171.6, 148.3, 147.1, 146.8, 140.8, 136.8, 130.4, 121.2. Anal. calcd. for $\mathrm{C}_{8} \mathrm{H}_{5} \mathrm{~N}_{4} \mathrm{O}_{3} \mathrm{Cl}: \mathrm{C}, 39.91$; H, 2.07; N, 23.28; Cl, 14.76. Found: C, 39.60; H, 2.00; N, 23.15; Cl, 14.50\%.

\section{Results and Discussion}

An environmentally benign synthetic method to produce 2-amino-5-substituted-1,3, 4-oxadiazoles would be highly attractive. We have synthesized 2-amino-5-substituted-1,3, 4-oxadiazole (1) derivatives through the electrooxidation of semicarbazone (4) at a platinum electrode in controlled potential electrolysis. The reaction sequences employed for the synthesis of title compound is shown in the Scheme 1. This electrochemical cyclization gives the oxadiazoles without requirement of any hazardous reagents. We have used acetic acid as a solvent and lithium perchlorate $\left(\mathrm{LiClO}_{4}\right)(0.001 \mathrm{M})$ as an electrolyte that can be handled very easily without major precautions.

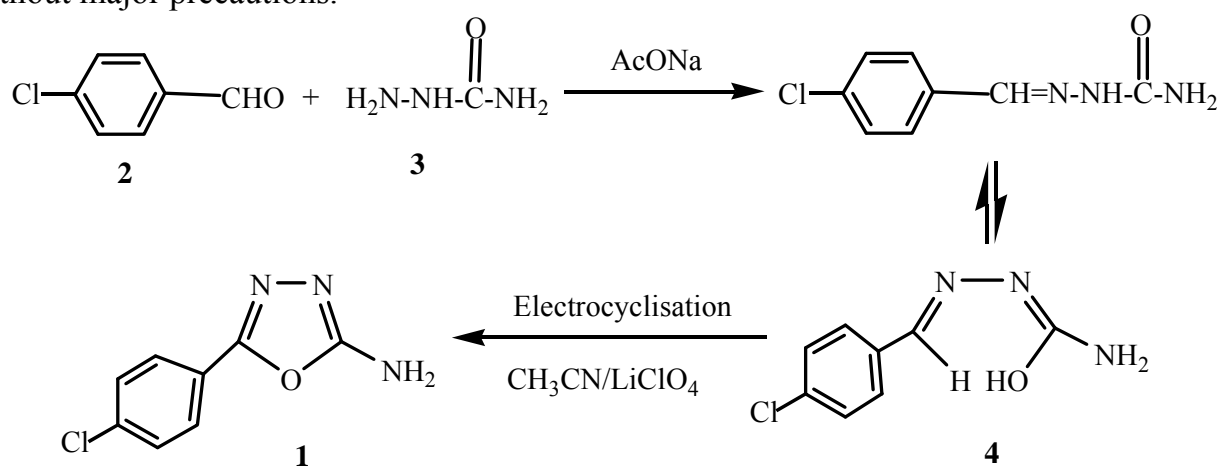

Scheme 1 


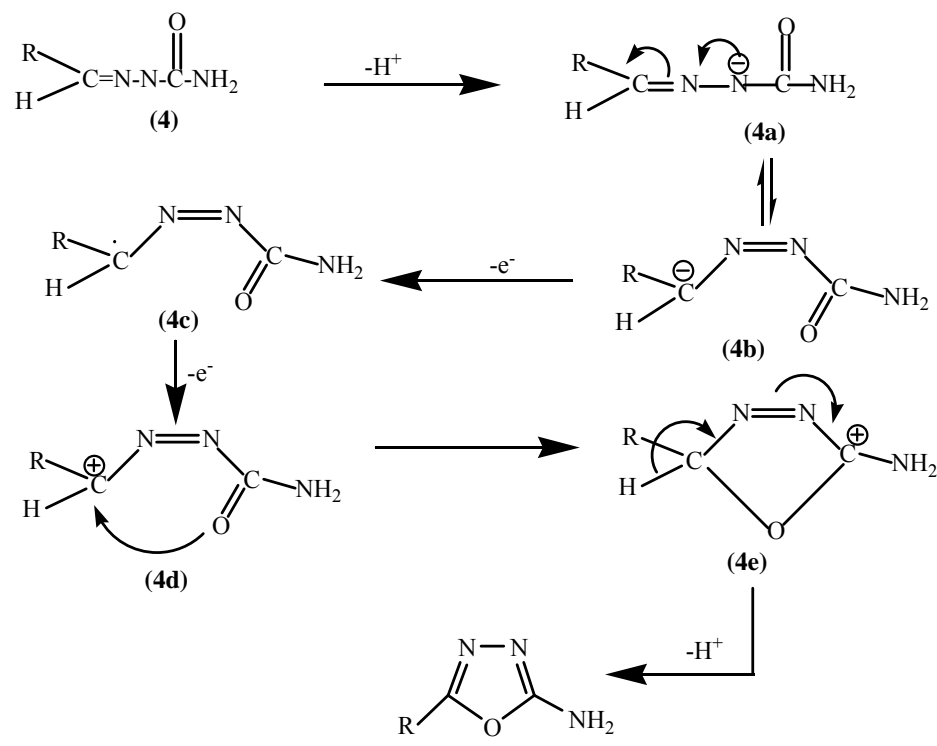

(1)

Scheme 2

However, to induce such cyclizations electrolytically has various merits. The electrochemical oxidation reactions do not require oxidizing chemicals because the oxidation takes place at the electrode and, furthermore, can be performed under mild conditions, e.g., at room temperature. Application of electricity as a non conventional energy source for activation of reactants in suitable solvents has now gained popularity over the usual homogeneous and heterogeneous reactions. It provides chemical processes with special attributes, such as enhanced reaction rate, higher yield of pure products, better selectivity and several ecofriendly advantages.

\section{Mechanistic proposals}

The first step as shown in Scheme 2 represents the deprotonation of (4) to form an anion (4a), which rearranges itself into (4b) and evolves a free radical (4c) after one electron oxidation. Subsequent second electron oxidation from the free radical (4c) gives a carbocation (4d). The formation of carbon oxygen bond completes the ring. On loosing a proton in the last step 2-amino-5-substituted-1,3,4-oxadiazole (1) is obtained.

2-Amino-5-substituted-1,3,4-oxadiazoles have been synthesized in excellent yields using the synthetic route outlined in Scheme 1. IR, ${ }^{1} \mathrm{H}$ NMR, ${ }^{13} \mathrm{C}$ NMR and mass spectral data are in good agreement with the proposed structures of all the synthesized compounds. Lack of ${ }^{1} \mathrm{H}$ NMR resonances observed with $\mathrm{NH}$ and $\mathrm{NH}_{2}$ functions in the ${ }^{1} \mathrm{H}$ NMR spectrum of (1a-n) proved that ring closure starting from (4) resulted in the formation of 1,3,4-oxadiazole ring. This was further substantiated by the ${ }^{13} \mathrm{C}$ NMR data of (1) which showed the peaks at $\delta 170-173$ and 145-150 due to $C_{2}$ and $C_{5}$ of oxadiazole respectively. Infra red spectrum shows $1600-1620 \mathrm{~cm}^{-1}$ for $(\mathrm{C}=\mathrm{N}-\mathrm{N}=\mathrm{C})$ and $1063-1073 \mathrm{~cm}^{-1}$ for $(\mathrm{C}-\mathrm{O}-\mathrm{C})$ in the compounds (1a-n) which confirmed the synthesis of 1,3,4-oxadiazoles.

\section{Conclusion}

In conclusion we have developed a convenient method for the synthesis of novel 2-amino-5substituted-1,3,4-oxadiazole derivatives in excellent yields which is not so easy to achieve by 
chemical methods. In the present electrolytic method, electrolysis was carried out at ordinary temperature and no hazardous chemicals were used. Therefore the method is environmentally benign and the great contribution in the field of green chemistry.

\section{Acknowledgment}

The author greatfully acknowledge the financial assistance provided by University Grant Commission, New Delhi and to the Sophisticated Analytical Instrumentation Facility (SAIF), A division of CDRI (Central Drug Research Institute) Lucknow, for providing spectra and microanalyses.

\section{References}

1. Holla B S, Gonaslaves R and Shenoy S, Eur J Med Chem., 2000, 35, 267-271.

2. Cesur N, Birteksoz S and Otuk G, Acta Pharm Turcica., 2002, 44, 23.

3. Laddi U V, Desai S R, Bennur R S and Bennur S C, Indian J Heterocycl Chem., 2002, 11, 319-322.

4. Zou X, Zhang Z and Jin G, J Chem Res Synopses, 2002, 228-230.

5. Zou X J, Lai L-H, Jin G-Y and Zhang Z X, J Agric Food Chem., 2002, 50, 3757-3760.

6. Palaska E, Sahin G, Kelicen P, Durlu N T and Altinok G, Farmaco, 2002, 57, 101-107.

7. Tyagi M and Kumar A, Oriental J Chem., 2002, 18, 125.

8. Clapp L B, Katritzky A R and Rees C W, Comprehensive Heterocyclic Chemistry, Pergamon Press, Oxford, 1984, p. 365.

9. Afiatpour P, Srivastava R M, Oliveira M L and Barreiro E J, Braz J Med Biol Res., 1994, 27, 1403.

10. Ley S V, Baxendale I R, Bream R N, Jackson P S, Leach A G, Longbottom D A, Nesi M, Scott J S, Storer R I and Taylor S J, J Chem Soc Perkin Trans I, 2000, 3815.

11. Adams G L, Graybill T L, Sanchez R M, Magaard V W, Burton G and Rivero R A, Tetrahedron Lett., 2003, 44, 5041-5045.

12. Hetzheim A and Moeckel K, Adv Heterocycl Chem., 1967, 7, 183-224.

13. Hill J, Comprehensive Heterocyclic Chemistry, Edited by Potts K T, Pergamon Press, Oxford, 1984, 6, 427-446.

14. Chiba T and Okimoto M, J Org Chem., 1992, 57, 1375-1379.

15. Butler R N, Scott F L and O'Mahony T A F, Chem Rev., 1973, 73, 93.

16. Aboulwafa O M and Omar O M M E, Sulfur Lett., 1992, 14, 181-188.

17. Omar F A, Mahfouz N M and Rahman M A, Eur J Med Chem., 1996, 31, 819-825

18. Golovlyova S M, Moskvichev Y A, Alov E M, Kobylinskey D B and Ermolaeva V V, Chem Heterocycl Compd., 2001, 37, 1102-1706.

19. Liu F, Wang B and Zhang Z, Youji Нuaxue., 2001, 21, 1126-1131.

20. Gani R S, Pujar S S and Gadaginamath G S, Indian J Heterocycl Chem., 2002, 12, 25.

21. Wang X, Li Z, Wei B and Yang J, Synth Commun., 2002, 32, 1097-1103.

22. Brain C T and Brunton S A, Synlett., 2001, 382-384.

23. Brain C T, Paul J M, Loong Y and Oakley P J, Tetrahedron Lett., 1999, 40, 3275.

24. Faidallah H M, Sharshira E M, Basaif S A and A-Ba-Oum A E, Phosphorus Sulfur Silicon and the Related Elements, 2002, 177, 67-79.

25. Cappo F T, Evans K A, Graybill T L and Burton G, Tetrahedron Lett., 2004, 45, 3257-3260.

26. Shono T, Electroorganic Synthesis, Academic Press, London, 1991.

27. Mann C K and Barnes K K, Electrochemical Reactions In Nonaqueous Systems, Marcel Dekker, Inc. New York, 1970.

28. Fry A J, Synthetic Organic Electrochemistry, $2^{\text {nd }}$ Ed., Wiley-Interscience Publication, New York, 1989. 


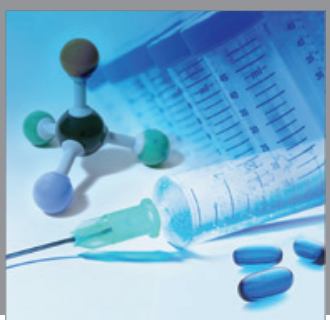

International Journal of

Medicinal Chemistry

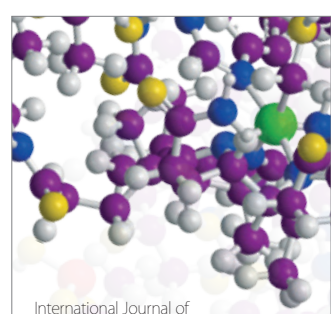

Carbohydrate Chemistry

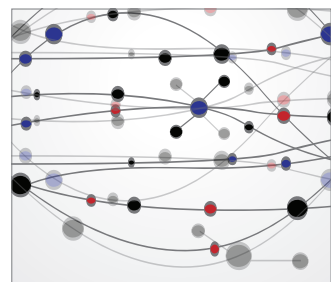

The Scientific World Journal
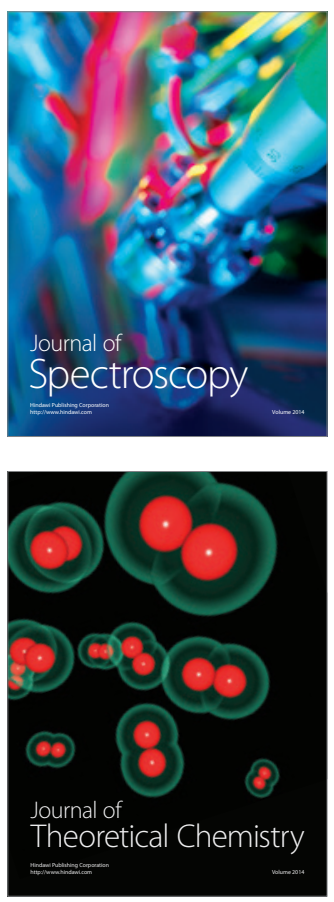
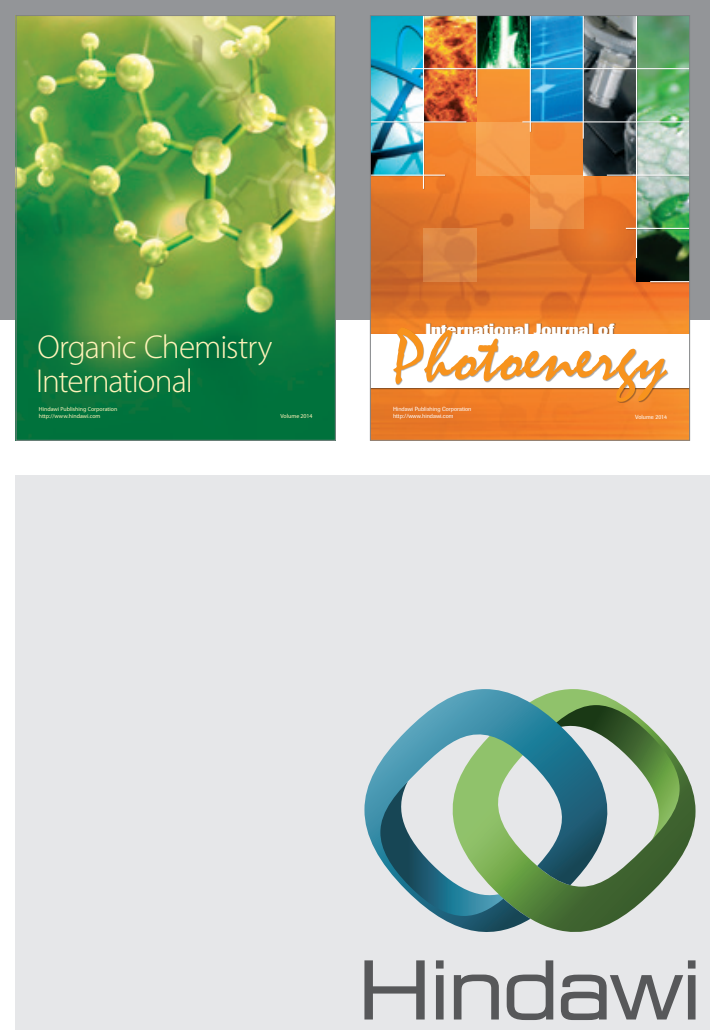

Submit your manuscripts at

http://www.hindawi.com
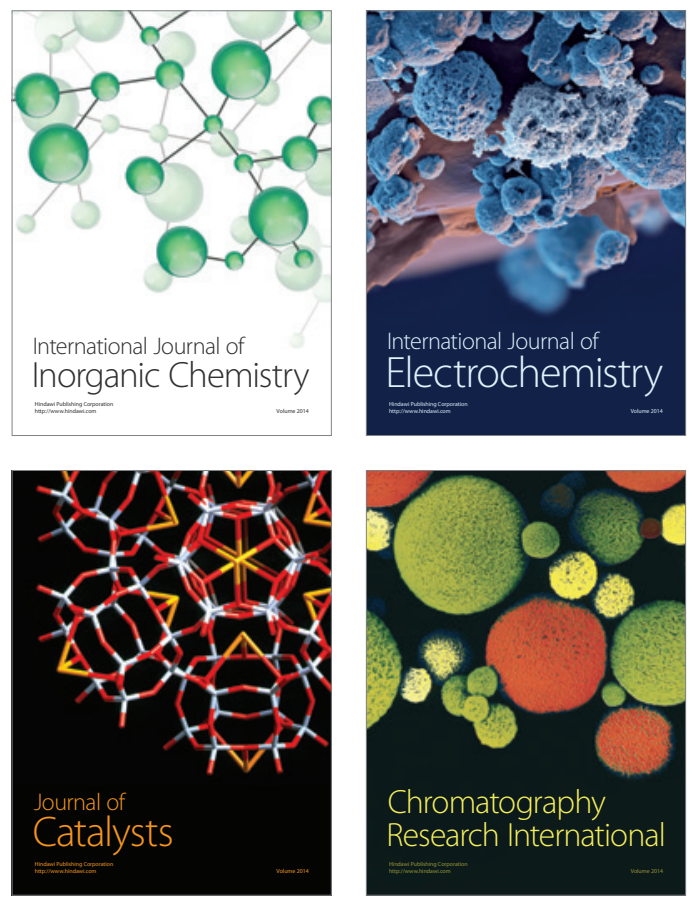
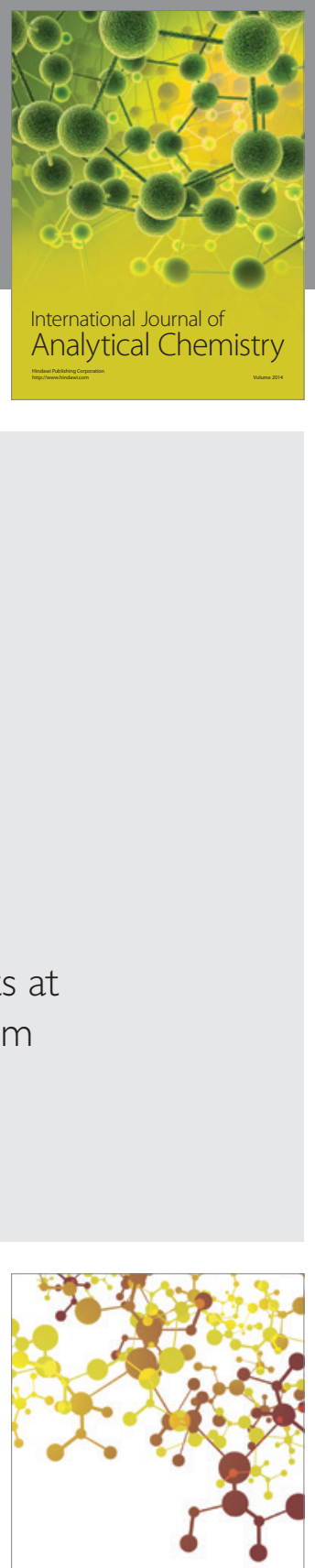

Journal of

Applied Chemistry
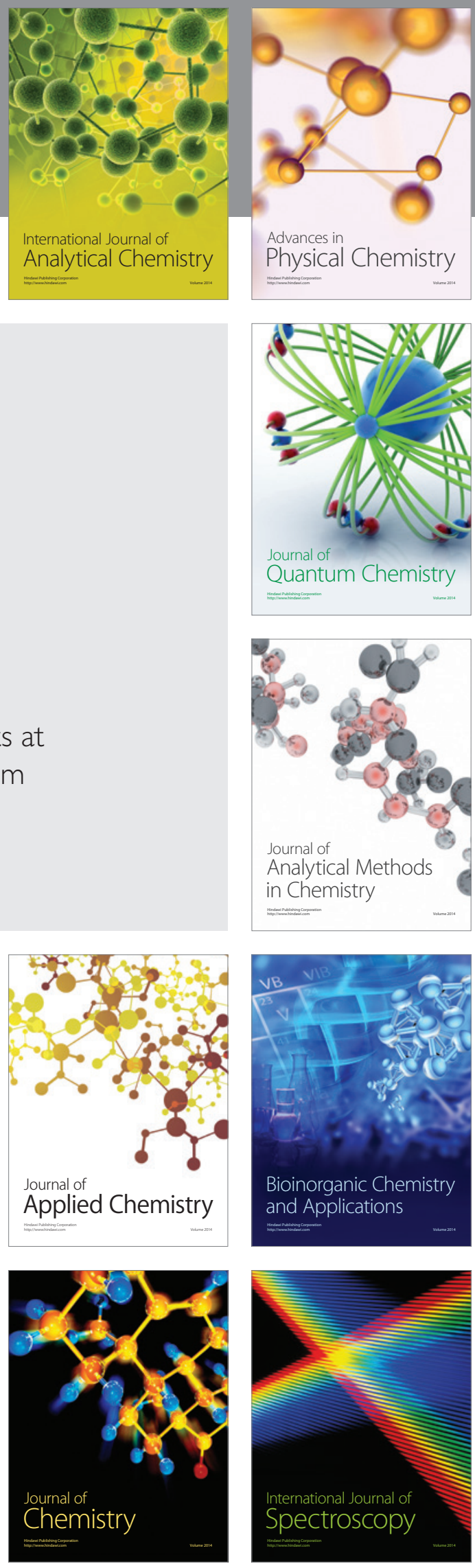